\title{
ATIVIDADE MICROBIANA DO SOLO EM SISTEMAS AGROFLORESTAIS, MONOCULTURAS, MATA NATURAL E ÁREA DESMATADA ${ }^{1}$
}

\author{
Sebastião Lourenço de Assis Júnior ${ }^{2}$, José Cola Zanuncio ${ }^{3}$, Maria Catarina Megumi Kasuya ${ }^{4}$, Laércio Couto ${ }^{5}$ e \\ Raul C.N. Melido ${ }^{6}$
}

\begin{abstract}
RESUMO - Este trabalho foi desenvolvido na Unidade Agroflorestal da Companhia Mineira de Metais (CMM), em Vazante, Estado de Minas Gerais, com o objetivo de estudar a atividade microbiana em diferentes ecossistemas, como sistemas agroflorestais (SAFs), monoculturas, área desmatada e mata natural, utilizando os métodos da respirometria no laboratório e da medida não-instantânea da taxa de evolução de $\mathrm{CO}_{2}$ no campo. A atividade biológica apresentou valores acumulados das taxas de respiração do solo, aos 20 dias, de 3,56 a 6,03 meq C-CO $2 / 100 \mathrm{~g}$ de solo no laboratório e de 165,16 a 559,37 $\mathrm{mg} \mathrm{CO}_{2} / \mathrm{m}^{-2}$.h no campo, na área desmatada e na mata nativa, respectivamente. Em ambos os ensaios, a atividade foi maior na mata nativa e nas pastagens, tanto em monocultivo quanto em SAFs. A atividade microbiana foi maior em SAF com arroz e eucalipto do que em arroz em monocultivo, pelo método no campo.
\end{abstract}

Palavras-chave: Sistemas agroflorestais, monoculturas e respiração do solo.

\section{SOIL MICROBIAL ACTIVITY IN AGROFOREST, MONOCULTURES, NATURAL FOREST AND DEFORESTED AREA SYSTEMS}

\begin{abstract}
This research was developed in the Agroforestry Unit of the "Companhia Mineira de Metais (CMM)", Vazante, Minas Gerais, Brazil, to study microbial activity in different ecosystems such as agroforests, monocultures, deforested areas and native forests using the method of respirometry under laboratory conditions and $\mathrm{CO}_{2}$ evaluation rate evolution under field conditions. Biological activity showed accumulated values of respiration rates at 20 days of 3.56 to $6.03 \mathrm{meq} C-\mathrm{CO}_{2} / 100 \mathrm{~g}$ of soil in the laboratory, and 165.16 to $559.37 \mathrm{mg} \mathrm{CO} / \mathrm{m}^{-2} . \mathrm{h}$ in the field, in deforested area and native forest, respectively. Activity was higher in native forest and pasture than in monoculture or agroforestry, in both experiments. Microbial activity was higher in agroforestry with rice and eucalypt than with rice in monoculture, under field conditions.
\end{abstract}

Key words: Agroforestry, monocultures, and soil respiration.

\section{INTRODUÇÃO}

Os sistemas agroflorestais (SAFs) são uma combinação integrada de árvores, arbustos, cultivos agrícolas e, ou, animais na mesma área, de maneira simultânea ou seqüencial, que buscam a otimização da agregação de valores socioeconômico-culturais e ambientais, com potencial para constituírem uma modalidade sustentável de uso e manejo dos recursos naturais. Embora esses sistemas apresentem várias vantagens em relação aos monocultivos (Mac Dicken \& Vergara, 1990), poucos são os trabalhos que as comprovam, especialmente no campo.

1 Recebido para publicação em 6.4.2001.

Aceito para publicação em 19.2.2003.

2 Faculdades Federais Integradas de Diamantina - FAFEID, Rua da Glória 187, 39100-000 Diamantina-MG; ${ }^{3}$ Departamento de Biologia Animal da Universidade Federal de Viçosa - UFV, 36571-000 Viçosa-MG; ${ }^{4}$ Departamento de Microbiologia da UFV; ${ }^{5}$ Departamento de Engenharia Florestal da UFV. ${ }^{6}$ Companhia Mineira de Metais, Fazenda Bom Sucesso, 38780-000 Vazante-MG. 
Vários parâmetros microbiológicos têm sido usados como indicadores para avaliar esses processos no solo, mas nenhum é adequado a todas as situações, devido à natureza dinâmica e complexa desses ecossistemas (Dick, 1992). Um bom indicador necessita responder às perturbações do solo, avaliar com precisão o funcionamento do sistema produtivo, indicar diferenças espaciais e temporais e ser relativamente rápido e de baixo custo (Turco et al., 1994).

Atributos químicos e físicos eram usados para definir a qualidade do solo em virtude da grande dificuldade de identificação de seus organismos, que podem apresentar mais de 10.000 espécies por grama (Torsvik et al., 1990) e representar cerca de 85\% da biomassa ou $90 \%$ do fluxo de $\mathrm{CO}_{2}$ entre componentes bióticos responsáveis pela decomposição de serapilheira em florestas decíduas (Paoletti \& Bressan, 1996).

A biomassa microbiana desempenha papel de destaque no cenário da sustentabilidade ambiental e pode ser estimada por métodos relativamente simples, pela quantificação de componentes extraídos do solo. A medida da taxa respiratória ou atividade microbiana, determinada pela evolução de $\mathrm{CO}_{2}$ oriundo da respiração de microrganismos heterotróficos aeróbicos durante a oxidação de compostos orgânicos, é uma das mais utilizadas (Kennedy \& Smith, 1995).

As avaliações da respiração microbiana em laboratório têm a vantagem de eliminar organismos da mesofauna e partes da planta que poderiam mascarar as avaliações (Kelting et al., 1998). Além disto, a temperatura e a umidade podem ser facilmente controladas, o que permite, inclusive, simulações. No entanto, quando amostras de solo são levadas ao laboratório, o grau de manipulação influencia a estrutura do solo e as trocas gasosas. No campo, as mensurações da respiração do solo consideram a serapilheira e são amplamente usadas para avaliar a atividade geral da biomassa no solo, sob a influência do clima, das propriedades físicas e químicas e de práticas agrícolas ou silviculturais, como rotação de culturas (Paul et al., 1999), adição de matéria orgânica (Schnürer et al., 1985) ou cobertura morta (Schroth et al., 1992), plantio direto (Balota et al., 1997), cultivo mínimo (Gunapala \& Scow, 1998), idade do povoamento, tipo de solo e espécies de árvores (Bauhus et al., 1998), mistura de espécies (Kautz \& Topp, 1998) e presença de animais e excrementos no solo (Bardget et al., 1998), que normalmente aumentam a biomassa e a atividade da microbiota do solo. O cultivo, além de afetar a química e

R. Árvore, Viçosa-MG, v.27, n.1, p.35-41, 2003 a estrutura do solo, diminui a atividade biológica devido à redução de macroagregados, que fornecem importantes microhabitats para a atividade microbiana (Dick, 1992).

A inclusão de componentes arbóreos pode manter ou aumentar a produtividade de determinado local, devido a processos que aumentem a entrada ou reduzem perdas no solo, como matéria orgânica, nutrientes e água, além de melhorar as propriedades físicas e químicas e beneficiar processos microbiológicos do solo (Young, 1994).

O objetivo deste trabalho foi estudar a atividade microbiana em diferentes ecossistemas, como sistemas agroflorestais (SAFs), monoculturas, área desmatada e mata natural, utilizando os métodos da respirometria no laboratório e da medida não-instantânea da taxa de evolução de $\mathrm{CO}_{2}$ no campo.

\section{MATERIAL E MÉTODOS}

Os estudos foram desenvolvidos em março de 1998 (laboratório) e janeiro de 1999 (campo), na Unidade Agroflorestal da Companhia Mineira de Metais (CMM), em Vazante, Estado de Minas Gerais (17 $36^{\circ}$ S, $46^{\circ} 42^{\prime}$ W). A precipitação anual, mínima e máxima da região foi 1.300 e $1.800 \mathrm{~mm}$, respectivamente, concentrada de novembro a março, o que gera um déficit hídrico anual de $171 \mathrm{~mm}$. As temperaturas médias máxima e mínima foram de 32 e $16^{\circ} \mathrm{C}$, respectivamente. A formação vegetal é o cerrado, com variações de campo limpo até matas (cerradão), e o tipo de solo predominante é o Latossolo Vermelho distrófico ( $\mathrm{LVd}$ ), com textura argilosa (CMM, comunicação pessoal).

Os dados foram obtidos de SAFs do tipo Alley cropping, constituindo piquetes de $500 \times 700 \mathrm{~m}$, com a espécie arbórea Eucalyptus camaldulensis em arranjo espacial de $10 \times 4 \mathrm{~m}$ e arranjo temporal seqüencial com arroz (ano zero), semeado na ocasião de sua implantação, seguido de soja (ano um), milho (ano dois) e pastagens com gado (do ano três até a rotação da floresta); e monocultivos dos componentes envolvidos nos consórcios (área superior aos piquetes), inclusive o eucalipto com e sem sub-bosque, mata nativa e área desmatada. Assim, o experimento constou de dez sistemas de uso da terra: monoculturas de soja, milho e pastagens; SAFs compostos por eucalipto + soja (eucasoja), eucalipto + milho (eucamilho) e eucalipto + pastagens (eucapim); eucalipto sem sub-bosque; eucalipto com sub-bosque (ecabosque); e área desmatada e mata nativa. 
Em todos os sistemas foram coletadas amostras de solo compostas por 12 subamostras simples de aproximadamente $100 \mathrm{~g}$ cada uma, obtidas até uma profundidade de $10 \mathrm{~cm}$. Ainda no campo, as amostras foram passadas por uma peneira de $2 \mathrm{~mm}$ de malha, acondicionadas em sacos plásticos e levadas ao Laboratório de Associações Micorrízicas do Instituto de Biotecnologia Aplicado à Agropecuária (BIOAGRO) na Universidade Federal de Viçosa, em Viçosa, Estado de Minas Gerais, para avaliação da atividade respiratória da microbiota pelo método de quantificação de $\mathrm{CO}_{2}$ desprendido, sem adição de substrato.

Inicialmente foi determinada a capacidade de retenção de água pelo solo, através do método do funil. Em seguida, novas amostras de $100 \mathrm{~g}$ de solo seco ao ar foram distribuídas em erlenmeyers de $250 \mathrm{ml}$, adicionando-se água até que a umidade atingisse $60 \%$ da capacidade de campo. Duas amostras de cada tipo de uso da terra foram submetidas a um sistema hermeticamente fechado com fluxo de ar isento de $\mathrm{CO}_{2}$, através de um respirômetro, regulado por um timer para funcionar por 1 hora, a cada 4 horas, durante 20 dias. O fluxo foi ajustado em 15 a 20 bolhas por minuto, direcionado a tubos que continham $20 \mathrm{ml}$ de solução de $\mathrm{NaOH} 0,5 \mathrm{~N}$ (armadilha), que retém o $\mathrm{CO}_{2}$ resultante da atividade biológica, formando o $\mathrm{Na}_{2} \mathrm{CO}_{3}$ em solução. Após 24, 72, $120,168,240,336$ e 480 horas, os tubos foram retirados, vedados com rolhas de borracha e substituídos por novos tubos, com a mesma solução do alcali. Desses tubos, foram coletadas duas alíquotas a $5 \mathrm{ml} \mathrm{em}$ cada intervalo de tempo, as quais foram submetidas à titulação com $\mathrm{HCl}$ $0,5 \mathrm{~N}$, com duas gotas de solução alcoólica de fenolftaleína como indicador e $10 \mathrm{ml}$ de solução de $\mathrm{BaCl}_{2}$ $0,2 \mathrm{~N}$, para precipitação do carbonato e evitar sua interferência na titulação. Foram utilizados dois frascos branco (controle) para descontar o $\mathrm{CO}_{2}$ que poderia, ainda, estar no sistema. Desta forma, a quantidade de $\mathrm{CO}_{2}$ desprendida de cada amostra foi obtida ao titular o alcali residual ou não-reativo, pois todo esse gás é removido sob a forma de $\mathrm{BaCO}_{3}$. Os resultados em meq de $\mathrm{CO}_{2}$ por $100 \mathrm{~g}$ de solo foram obtidos pelo cálculo da taxa respiratória (TR), dada por: $\mathrm{TR}=\mathrm{N} \times \mathrm{f} \times(\mathrm{Vb}-\mathrm{Va}) \times 20 / 5$, em que $\mathrm{N}=$ normalidade do $\mathrm{HCl} ; \mathrm{f}=$ fator de correção da normalidade do $\mathrm{HCl} ; \mathrm{Vb}=$ volume $(\mathrm{ml})$ de $\mathrm{HCl}$ gasto para titular o branco; $\mathrm{Va}=$ volume $(\mathrm{ml})$ de $\mathrm{HCl}$ gasto para titular o $\mathrm{NaOH}$ correspondente às amostras de solo; $20=$ volume (ml) de $\mathrm{NaOH}$ nos tubos; e $5=$ volume $(\mathrm{ml})$ de $\mathrm{NaOH}$ usado em cada titulação.
Em cada sistema foram montadas cinco campânulas, sendo uma controle (branco), introduzida no solo até uma profundidade de $2 \mathrm{~cm}$. As campânulas, de $18 \mathrm{~cm}$ de diâmetro e $18 \mathrm{~cm}$ de altura, foram cobertas com papel alumínio, com a finalidade de refletir melhor as radiações solares. Como o $\mathrm{CO}_{2}$ evolui da superfície do solo, ele é retido nas campânulas até ficar difuso e ser absorvido pelo álcali ( $70 \mathrm{ml}$ de $\mathrm{NaOH} 0,5 \mathrm{~N}$ ) em potes de vidro de $250 \mathrm{ml}$, dispostos sobre tripés metálicos a $2 \mathrm{~cm}$ do solo. Nas campânulas-controle, o álcali permaneceu fechado nos frascos. Depois de montadas, as campânulas foram protegidas por coberturas de madeira, para evitar a incidência direta de raios solares, e deixadas no campo por aproximadamente 24 horas, anotando-se a hora de instalação e de desmontagem, para obter o tempo de exposição do álcali ao $\mathrm{CO}_{2}$. Após o período de exposição, as campânulas foram retiradas, os frascos tampados e hermeticamente vedados com cola de silicone e, então, levados ao laboratório para análise, pelo método titulométrico, descrito com o seguinte cálculo: $\mathrm{TR}=\mathrm{N}$ x f x $(\mathrm{Vb}-\mathrm{Va})$ x $22 \times 70 / 5 \times 0,025^{-1} \times \mathrm{T}^{-1}$, em que $22=$ equivalente grama do $\mathrm{CO}_{2} ; 70=$ volume $(\mathrm{ml})$ de $\mathrm{NaOH}$ nos frascos, $5=$ volume $(\mathrm{ml})$ de $\mathrm{NaOH}$ usado para cada titulação, 0,025 = área das campânulas em $\mathrm{m}^{2}$; e $\mathrm{T}=$ tempo de exposição do álcali ao $\mathrm{CO}_{2}$, expresso em horas.

As medidas das taxas respiratórias no laboratório e no campo foram submetidas aos testes de Lilliefors, para verificar se seguiam a distribuição normal, e de Bartlett, para verificar a homogeneidade de variâncias. Em seguida, estas foram submetidas à análise de variância e as médias comparadas pelo teste de agrupamento de ScottKnott, a 5\% de significância.

\section{RESULTADOS E DISCUSSÃO}

\subsection{Atividade da Microbiota no Laboratório}

A atividade biológica, avaliada pela quantidade total de $\mathrm{CO}_{2}$ desprendido durante o processo de decomposição da matéria orgânica em respirômetro, no laboratório, apresentou valores acumulados de 3,56 a $6,03 \mathrm{meq} \mathrm{C}-\mathrm{CO}_{2} / 100 \mathrm{~g}$ de solo para as taxas de respiração do solo aos 20 dias, para a área desmatada e a mata nativa, respectivamente (Figura 1).

No $20^{\circ}$ dia, os sistemas mata e pastagem em monocultura e consorciada com eucalipto com $6,03,5,28$ e $5,08 \mathrm{meq} \mathrm{C}-\mathrm{CO}_{2} / 100 \mathrm{~g}$ de solo, respectivamente, apresentaram maior atividade biológica que os demais usos da

R. Árvore, Viçosa-MG, v.27, n.1, p.35-41, 2003 
terra. Esses resultados foram semelhantes aos obtidos por Fialho et al. (1991), quando investigaram a influência da cobertura vegetal sobre a atividade da microbiota do solo. De 0-2 cm de profundidade, os autores encontraram 6,61; 6,33 e 6,00 meq C-CO $/ 100 \mathrm{~g}$ de solo na mata, na pastagem e no eucalipto, respectivamente, concluindo que os maiores teores de nutrientes e valores de $\mathrm{pH}$ na mata $\mathrm{e}$ os maiores teores de alumínio trocável e acidez titulável no eucalipto foram os fatores responsáveis por essas diferenças. Della Bruna et al. (1991) e Carvalho et al. (1997) também encontraram maior atividade sob a mata do que no eucalipto e relataram que solos sob eucalipto apresentam menor teor de bases e maior relação carbono/ nitrogênio, o que contribui para explicar menores atividades nesses sistemas.

Os sistemas com pastagens apresentaram altas taxas respiratórias, o que pode ser devido ao maior acúmulo de matéria orgânica no solo sob essa cobertura vegetal, comumente semelhante ou maior que aquela sob floresta, como ocorre em solos na Amazônia (Feigl et al., 1997). A grande abundância de fezes bovinas contribui para esse fato, pois a presença de animais e a adição de excrementos no solo promoveram aumento significativo da biomassa de microrganismos. Tal aumento tem papel importante no fornecimento de substrato adicional para o metabolismo e crescimento microbiano, o que, conseqüentemente, altera a disponibilidade de nutrientes no solo.

Mallik \& Hu (1997), Janssens et al. (1998) e Lytle \& Cronan (1998) revelaram que sistemas submetidos a corte raso podem apresentar taxas respiratórias do solo mais altas que florestas intactas. A área desmatada, no entanto, apresentou baixa atividade biológica, de forma semelhante ao relatado por Striegl \& Wickland (1998), o que pode ser devido ao fato de esta área ter sido desmatada com correntão, prática que destrói grande número de raízes, o que não ocorre com corte raso.

Não houve diferença significativa entre os SAFs e seus componentes não-arbóreos em monocultura, de forma semelhante ao registrado para eucalipto com e sem sub-bosque, o que evidencia que o uso desse procedimento metodológico seja ineficiente para avaliar a atividade biológica na profundidade utilizada.

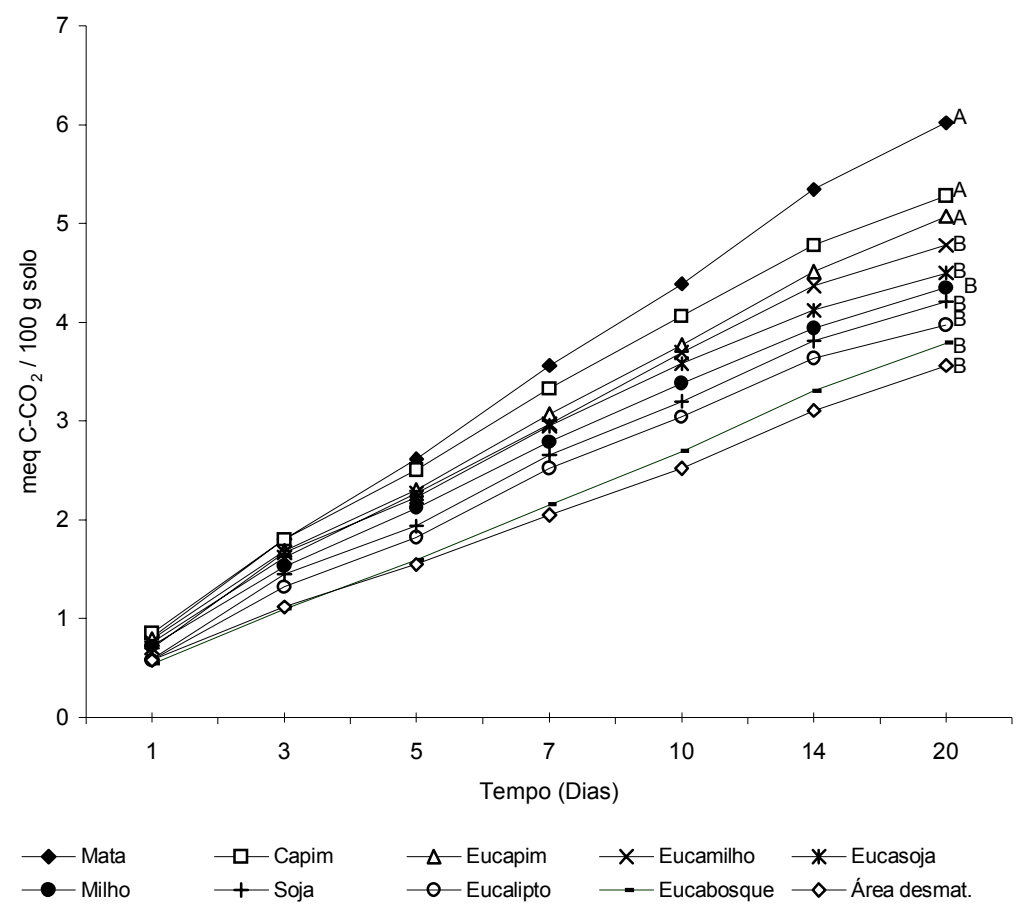

Médias seguidas de mesma letra não diferem entre si pelo teste de Scott-Knott, a 5\% de probabilidade.

Figura 1 - Evolução de $\mathrm{CO}_{2}$ em meq C-CO $/ 100 \mathrm{~g}$ de solo aos 20 dias, resultante da atividade biológica em diferentes sistemas de uso da terra, na profundidade de 0-10 cm, em Vazante, Minas Gerais, Brasil.

Figure 1 - Evolution of $\mathrm{CO}_{2}$ (meq $\mathrm{C}$ - $\mathrm{CO}_{2} / 100 \mathrm{~g}$ of soil) at twenty days, resulting from biological activity under differing land use systems, 0-10 cm deep. Vazante, Minas Gerais, Brazil.

R. Árvore, Viçosa-MG, v.27, n.1, p.35-41, 2003 
Della Bruna et al. (1991) mostraram que a adição de serapilheira pode aumentar cerca de cinco vezes a atividade biológica nos solos, o que indica que a adição de uma fonte de carbono pode ser uma alternativa eficaz para aumentar a sensibilidade desse método. Esse procedimento metodológico, mesmo com a adição de $1 \mathrm{~g}$ de glicose por amostra de solo, mostrou diferenças reduzidas para a atividade da microbiota do solo sob diferentes coberturas vegetais, o que indica que a maior disponibilidade de carbono e nutrientes em camadas superficiais, independentemente da cobertura vegetal, pode ser responsável por tais diferenças, que são mais nítidas em camadas mais profundas, apesar de mais baixas (Franco et al., 1994). Este fato motivou a confirmação dos resultados no campo, onde a camada de serapilheira é mantida em sua estrutura original.

\subsection{Atividade da Microbiota no Campo}

A atividade biológica não-instantânea, avaliada pela quantidade total de $\mathrm{CO}_{2}$ desprendido durante o processo de decomposição da matéria orgânica no campo, apresentou taxas de respiração no solo entre 165,16 e $559,37 \mathrm{mg} \mathrm{CO} / \mathrm{m}^{2}$.h (Figura 2). Estes valores correspondem a $1.446,80$ e $4.900,08 \mathrm{~g} \mathrm{CO}_{2} / \mathrm{m}^{2}$.ano, sendo próximos aos encontrados por Wiant (1967), que variaram entre 1.800 e $6.100 \mathrm{~g} \mathrm{CO}_{2} / \mathrm{m}^{2}$.ano, taxas consideradas típicas para solos florestais (Edward \& Sollins, 1973).

Os sistemas mata nativa, com 559,37 $\mathrm{mg} \mathrm{CO} / \mathrm{m}^{2} . \mathrm{h}$, e pastagens em monocultura e consorciada com eucalipto, com 538,25 e $488,81 \mathrm{mg} \mathrm{CO} / \mathrm{m}^{2} . h$, respectivamente, apresentaram maior atividade biológica, enquanto os menores valores foram registrados no arroz e na área desmatada com 202,82 e $165,16 \mathrm{mg} \mathrm{CO}_{2} / \mathrm{m}^{2}$.h, respectivamente (Figura 2), confirmando os resultados de laboratório (Figura 1).

O valor encontrado para mata nativa foi maior que o de Schulze (1967) em savanas e floresta decídua seca, e inferior ao de florestas de galerias, florestas úmidas e matas secundárias durante uma estação seca na Costa Rica. Esses valores são coerentes, pois na ocasião da avaliação houve precipitação de $5 \mathrm{~mm}$. Rai \& Srivastava (1981) e Orchard \& Cook (1983) também mostraram que a precipitação afeta diretamente a umidade e a temperatura do solo, as quais têm influência na atividade da microbiota e verificaram relações lineares entre o potencial de água no solo e sua atividade biológica. Além disto, a respiração microbiana é influenciada pela rizosfera, com maior volume em solos florestais, onde pode atingir $20 \%$ da respiração total e ser fonte significante de C fixado fotossinteticamente nestes ecossistemas (Kelting et al., 1998).

Os altos valores da TR, em sistemas com pastagens solteira e consorciada com eucalipto, com 538,25 e $488,81 \mathrm{mg} \mathrm{CO} / \mathrm{m}^{2}$.h, respectivamente, podem ser devido à grande quantidade de biomassa microbiana acumulada na serapilheira sob essas coberturas vegetais, pois a biomassa microbiana pode atingir um valor quatro vezes maior em ecossistemas com pastagens que em solos sob floresta (Feigl et al. 1997). Essa diferença pode estar relacionada com a composição do material vegetal, indicando a análise de parâmetros qualitativos, como relação $\mathrm{C} / \mathrm{N} /$ P e índice ligno-celulósico. Costa (1995) mostrou valores de 396,17 e $356,33 \mathrm{mg} \mathrm{CO}_{2} / \mathrm{m}^{2}$.h em solos sem resíduos de exploração, em povoamentos de eucalipto no Espírito Santo, 12 meses após a colheita, em local sob leira e adjacente a esta, respectivamente com esse procedimento metodológico. Tal fato é semelhante ao obtido em sistemas com eucalipto como componente arbóreo, com valores de 283,84 $\mathrm{mg} \mathrm{CO}_{2} / \mathrm{m}^{2}$.h a 488,81 $\mathrm{mg} \mathrm{CO}_{2} / \mathrm{m}^{2}$.h em consórcio com arroz e pastagem, respectivamente.

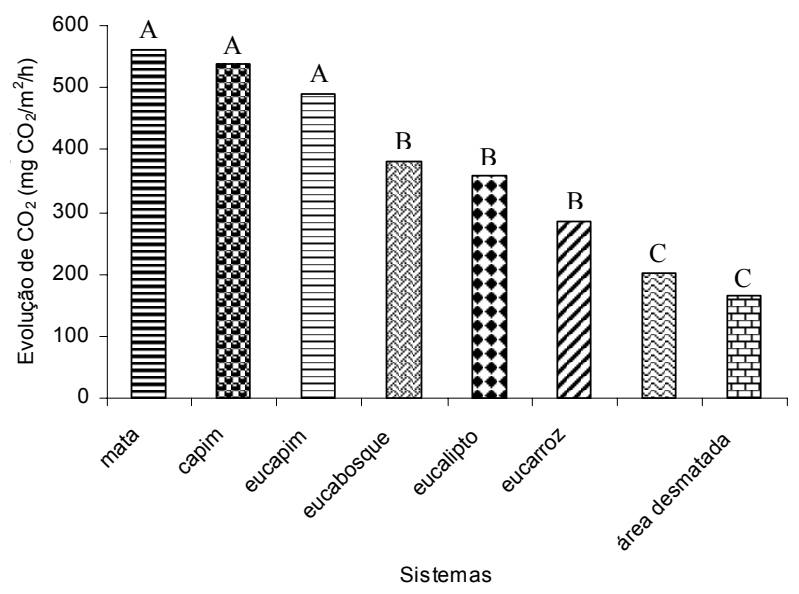

Médias seguidas de mesma letra não diferem entre si pelo teste de Scott-Knott, a 5\% de probabilidade.

Figura 2 - Evolução de $\mathrm{CO}_{2}\left(\mathrm{mg} \mathrm{CO}_{2} / \mathrm{m}^{2} \cdot \mathrm{h}\right)$ resultante da atividade biológica em diferentes sistemas de uso da terra, em Vazante, Minas Gerais, Brasil.

Figure 2 - Evolution of $\mathrm{CO}_{2}\left(\mathrm{mg} \mathrm{CO}_{2} / \mathrm{m}^{2} . h\right)$ resulting from biological activity under different systems of land use, Vazante, Minas Gerais, Brazil.

R. Árvore, Viçosa-MG, v.27, n.1, p.35-41, 2003 
Os dados referentes à área de soja em monocultura e consorciada com eucalipto foram descartados devido à interferência de formigas nos frascos armadilha com $\mathrm{NaOH}$. Por isto, recomenda-se a utilização de organza, que permite a entrada de ar e impede a contaminação do alcali por organismos da mesofauna.

Embora a heterogeneidade florística e a cobertura do solo possam minimizar a perda de nutrientes e proporcionar melhores condições para a atividade de microrganismos no solo, as taxas respiratórias nos sistemas eucalipto com e sem sub-bosque com 380,23 e $359,70 \mathrm{mg} \mathrm{CO}_{2} \mathrm{~m}^{-2} \mathrm{~h}^{-1}$, respectivamente, foram semelhantes. No entanto, esses valores foram maiores que nos sistemas mais simples, como arroz em monocultura e área desmatada, o que revela a maior sensibilidade do método no campo, por considerar a serapilheira que fora descartada no processo de peneiramento das amostras para o ensaio de laboratório. Além disto, a atividade biológica no sistema composto por arroz e eucalipto foi maior que aquela do sistema com arroz em monocultura, o que evidencia que o componente arbóreo exerce influência positiva nas condições microbiológicas do solo (Young, 1994).

Os métodos de laboratório e campo são adequados para o estudo da atividade microbiana, por respiração do solo. No entanto, o método de avaliação com medições não-instantâneas da atividade respiratória no campo mostrou-se importante para complementação dos resultados. Além disto, este método é simples, de baixo custo e apresenta maior sensibilidade por considerar as condições naturais.

\section{AGRADECIMENTO}

Ao Conselho Nacional de Desenvolvimento Científico e Tecnológico (CNPq), à Coordenação de Aperfeiçoamento de Pessoal de Nível Superior (CAPES), à Fundação de Amparo à Pesquisa do Estado de Minas Gerais (FAPEMIG) e à Companhia Mineira de Metais.

\section{REFERÊNCIAS BIBLIOGRÁFICAS}

BALOTA, E. L. et al. Atividade microbiana em sistemas de preparo do solo e sucessão de culturas. In: CONGRESSO BRASILEIRO DE CIÊNCIA DO SOLO, 26., Rio de Janeiro, 1997. Resumos expandidos... Rio de Janeiro: SBSC, 1997. CD - ROM.

R. Árvore, Viçosa-MG, v.27, n.1, p.35-41, 2003
BARDGETT, R. D. et al. Dynamic interactions between soil animals and microorganisms in upland grassland soils amended with sheep dung: a microcosm experiment. Soil Biology \& Biochemistry, v. 30, n. 4, p. 531-539, 1998.

BAUHUS, J.; PARÉ, D.; CÔTÉ, L. Effects of tree species, stand age and soil type on soil microbial biomass and its activity in a Southern Boreal forest. Soil Biology $\&$ Biochemistry, v. 30, n. 8, p. 1077-1089, 1998.

CARVALHO, M. C. S. et al. Atividade microbiana de um latossolo vermelho escuro álico sob eucalipto e mata nativa. In: CONGRESSO BRASILEIRO DE CIÊNCIA DO SOLO, 26., Rio de Janeiro, 1997. Resumos expandidos... Rio de Janeiro: SBSC, 1997. CD - ROM.

COSTA, M. D. Atividade biológica e liberação de nutrientes em resíduos da exploração de povoamentos de eucalipto. 1995. 91 f. Dissertação (Mestrado em Microbiologia) - Universidade Federal de Viçosa, ViçosaMG, 1995.

DELLA BRUNA, E. et al. Atividade da microbiota de solos adicionados de serapilheira de eucalipto e de nutrientes.

Revista Brasileira de Ciência do Solo, v. 15, p. 15-20, 1991.

DICK, R. P. A review: long-term effects of agricultural systems on soil biochemical and microbial parameters.

Agricultural Ecosystems Environmental, v. 40, p. 25-36, 1992.

EDWARDS, N. T.; SOLLINS, P. Continuous measurement of carbon dioxide evolution from partitioned forest floor components. Ecology, v. 54, n. 2, p. 406-412, 1973.

FEIGL, B. J.; FERNANDES, S. A. P.; RIZZO, R. T. Biomassa microbiana da serapilheira e do solo sob floresta e pastagem em Rondônia. In: CONGRESSO BRASILEIRO DE CIÊNCIA DO SOLO, 26., 1997, Rio de Janeiro. Resumos expandidos... Rio de Janeiro: SBSC, 1997. CD ROM.

FIALHO, J. F.; BORGES, A. C.; BARROS, N. F. Cobertura vegetal e as características químicas e físicas e atividade da microbiota de um latossolo vermelho-amarelo distrófico.

Revista Brasileira de Ciência do Solo, v. 15, p. 21-28, 1991.

FRANCO, F. S.; GJORUP, G. B.; CARVALHO, A. F. Avaliação de características físicas, químicas e microbiológicas de um solo sob sistema agroflorestal comparado com mata secundária e pastagem na região de Viçosa, MG. In: CONGRESSO BRASILEIRO SOBRE SISTEMAS AGROFLORESTAIS, 1., 1994, Porto Velho. Anais... Colombo: EMBRAPA-CNPF, 1994. p. 259. 
GUNAPALA, N.; SCOW, K. M. Dynamics of soil microbial biomass and activity in conventional and organic farming systems. Soil Biology \& Biochemistry, v. 30, n. 6, p. 805816, 1998.

JANSSENS, I. A.; BARIGAH, S. T.; CEULEMANS, R. Soil $\mathrm{CO}_{2}$ efflux rates in different tropical vegetation types in French Guiana. Annales des Sciences Forestieres, v. 55, n. 6, p. 671-680, 1998.

KAUTZ, G.; TOPP, W. Sustainable forest management for improving soil quality. Forstwissenschaftliches

Centralblatt, v. 117, n. 1, p. 23-43, 1998.

KELTING, D. L.; BURGER, J. A.; EDWARDS, G. S. Estimating root respiration, microbial respiration in rhizosphere, and root-free soil respiration in forest soils. Soil Biology \& Biochemistry, v. 30, n. 7, p. 961-968, 1998.

KENNEDY, A. C.; SMITH, K. L. Soil microbial diversity and the sustainability of agricultural soils. Plant and Soil, v. 170, p. $75-86,1995$.

LYTLE, D. E.; CRONAN, C. S. Comparative soil $\mathrm{CO}_{2}$ evolution, litter decay, and root dynamics in clearcut and uncut spruce-fir forest. Forest Ecology and Management, v. 103, n. 2/3, p. 121-128, 1998.

MacDICKEN, K.G.; VERGARA, N. T. (Eds.).

Agroforestry: classification and management. New York: John Wiley \& Sons, 1990. 382 p.

MALLIK, A. U.; HU, D. Soil respiration following site preparation treatments in boreal mixedwood forest. Forest Ecology and Management, v. 97, n. 3, p. 265-275, 1997.

ORCHARD, V.; COOK, F. J. Relationship between soil respiration and soil moisture. Soil Biology Biochemistry, v. 15, n. 4 , p. 447-453, 1983.

PAOLETTI, M. G.; BRESSAN, M. Soil invertebrates as bioindicators of human disturbance. Critical Reviews in Plant Sciences, v. 15, n. 1, p. 21-62, 1996.
PAUL, E. A. et al. Evolution of $\mathrm{CO}_{2}$ and soil carbon dynamics in biologically managed, row-crop agroecosystems. Applied Soil Ecology, v. 11, n. 1, p. 53$65,1999$.

RAI, B.; SRIVASTAVA, A. K. Studies on microbial population of a tropical dry deciduous forest soil in relation to soil respiration. Pedobiologia, v. 22, p. 185-190, 1981.

SCHNÜRER, J.; CLARHOLM, M.; ROSSWALL, T. Microbial biomass and activity in an agricultural soil with different organic matter contents. Soil Biology \& Biochemistry, v. 17, n. 5, p. 611-618, 1985.

SCHROTH, G.; ZECH, W.; HEIMANN, G. Mulch decomposition under agroforestry conditions in a sub-humid tropical savanna process and influence of perennial plants. Plant and Soil, v. 147, p. 1-11, 1992.

SCHULZE, E. D. Soil respiration of tropical types. Ecology, v. 48, p. 652-653, 1967.

STRIEGL, R. G.; WICKLAND, K. P. Effects of clear-cut harvest on soil respiration in a jack pine - lichen woodland. Canadian Journal of Forest Research, v. 28, n. 4, p. 534539, 1998.

TORSVIK, V. et al. Comparison of phenotypic diversity and DNA heterogeneity in a population of soil bacteria. Applied Environmental Microbiology, v. 56, p. 776-781, 1990.

TURCO, R. F.; KENNEDY, A. C.; JAWSON, M. D. Microbial indicators of soil quality. In: DORAN, J. W.; COLEMAN, D. C.; BEZDICEK, D. F.; STEWART, B. A. Defining soil quality for a sustainable environment. Madison: SSSA, 1994. p. 73-90.

WIANT Jr., H. V. Has the contribution of litter decay to forest "soil respiration" been overestimated? Journal of Forestry, v. 65, p. 408-409, 1967.

YOUNG, A. Agroforestry for soil conservation. 4.ed. Wallingford, CAB International, 1994. 276 p. 